\title{
Nuclear Power Renaissance Based on Engineered Micro-Nano-Nuclear Materials
}

\author{
Liviu Popa-Simil \\ LAAS-Los Alamos Academy of Sciences, Los Alamos, NM, USA \\ Email: laaos@laaos.org
}

How to cite this paper: Popa-Simil, L. (2021) Nuclear Power Renaissance Based on Engineered Micro-Nano-Nuclear Materials. Energy and Power Engineering, 13, 65-74.

https://doi.org/10.4236/epe.2020.134B007

Received: February 23, 2021

Accepted: April 27, 2021

Published: April 30, 2021

\begin{abstract}
Nuclear Power today is in stagnation with a fleet of 440 operational units, due to many drawback factors, as economics, safety, controllability and response time, security and waste management, which all together act as a deterrent to new reactor construction. If the present trend is followed, together with aging of many nuclear plants, by 2040 there will remain less than half of the actual reactors in operation, representing an accelerated decay of the industry. The idea of renaissance of nuclear power is more frequent, but this is not possible without the use of novel materials, based on nano-engineered structures. It is well known that Damascus swords were not possible without the use of Damascus steel, and so the next nuclear technology is not possible without the use of novel micro-nano nuclear materials, which finally dictates the performances of the nuclear structures built with them. As a first approach to modern technology, since 1980s, five types of nuclear materials, able to bring a leap forward in nuclear technology have been identified and studied, which are: 1) Micro-hetero structures able to deal with fission products, that use fission reaction kinematics to self-separate fission products from the nuclear fuel, generically called "Cer-Liq-Mesh", because simply it consists of a ceramic material stabilized on an elastic mesh or felt, immersed into a drain liquid. This improves the radiation damage, fuel burnup, fission products separation, and specific power density. 2) Nano-Beaded-Hetero-Structures that are using the nano-cluster specific mechanisms to accelerate separation of the transmutation products and place them into a drain liquid, which improves the separation of minor actinides, and radioisotopes production. 3) $\mathrm{Na}$ no-hetero structures for direct nuclear energy conversion into electricity, that are resembling a supercapacitor, charged by the moving nuclear particles, and discharges delivering electricity, where the structure is made of repetitive conductive and insulating layers, generically known as "CIci", some of the variants creating hyperbolic metamaterials, that may deliver electricity and radiation. Using these structures, one may eliminate the thermos-mechanical
\end{abstract}


stage from the actual nuclear-thermo-mechano-electric energy conversion cycle, reducing it at nuclear-electric only and reducing the size of nuclear-electric plant by $90 \%$, creating a fission battery. 4) Radiation damage self-repairing materials made of a "fractal", multi-material interlaced structure that maintains its properties constant independent of radiation dose. These materials will be used for cladding and structures allowing a near-perfect burning, using breed \& burn technology. 5) Radiation guiding structures that are using nano-structures to trap and guide radiation on desired controllable path being used for control systems assuring a micro-second response time, and light shielding allowing the creation of mobile structures.

\section{Keywords}

Nano, Nuclear-Energy, Micro-Hetero Structure, Nano-Clustered-Hetero

Structure, Hetero-Nano-Structure, Nano, Energy Harvesting, Meta-Material' Advanced Power Generation, Energy Conversion, Safe Nuclear

\section{Introduction}

The Nuclear power as we know it today is a mature technology developed on the basis of 1950s materials, and the difficulties it encounters are deeply embedded in the material performances it now uses, which were developed asymptotically until it reached near maximum performances, where weight of new improvements is minimal. As is known today, in project management culture, that the electric light is not the result of continuous improvements in candle technology, that might be the case to nuclear power, where novel domains research branches as nano and hetero structures to be used to create new materials, where heterogeneity is "by design", which to possess exceptional properties [1], allow and drive towards nuclear structures product of a technological leap, as the fundament of the novel nuclear renaissance. Without these materials, nuclear renaissance remains inside the realm of dreams paved in nice words, and the nuclear fleet might become extinct by 2080s.

One of the problems is the accelerated degradation of nuclear fuel under the action of fission products, “ 0 " in Figure 1, transmutation products and radiation inside the active zone, corroborated with improper temperature distribution and mechanical stress induced during operation, that seems it cannot be solved properly using the actual materials.

Another problem was the production of minor actinides, “ $\underline{\underline{4}}$, that remain inside used fuel, problem solved in part by breed \& burn regimes, but we will also intend to have them together with other isotopes for other applications and their extraction from the present used fuel is difficult.

Another problem is related to the actual complexity of nuclear energy conversion that requires the use of thermos-mechanical followed by mechanical-electric cycle that ends in low conversion efficiency in the range of 30\%- 
Brief on: Other 4 Fundamental developments based on nano-technologies that can improve fission nuclear reactors

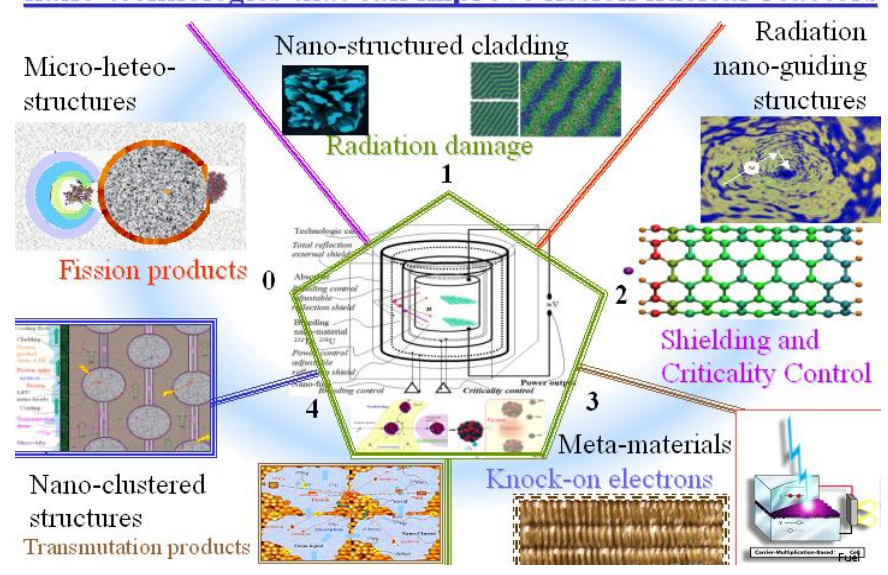

Figure 1. Engineered material classes for nuclear renaissance.

$50 \%$ and high complexity, which makes the "driving" of a nuclear reactor very intricate, " 3 ".

Following efficiently a daily electricity demand curve, is challenging and risky with actual reactors, because the actual reactivity (criticality) control system, that uses mainly the delayed neutrons, that represent a fraction of a percent from total neutrons, making the "nuclear reactor "driving", by about one order of magnitude more difficult than driving a car on an icy road. In order to improve this aspect one has to improve the actual mechanical-electric control system, based on absorption rods or reflective blankets, but needs such materials that to react in micro-second time frame to neutron flux variation, “ 2 ".

Nuclear fuel burnup and breed\&burn procedures are limited by the structural materials and cladding radiation damage low robustness, requiring periodical recladding and fuel refurbishment, that makes the process more complex and unpractical, " 1 ".

All these problems and few more may be solved by novel developed nuclear materials based on engineered micro-nano-heterogenous structures, which creates the basis for a real, reliable and credible nuclear renaissance.

\section{Micro-Hetero Structures}

The searches to solve nuclear fuel damage problems conducted to development of micro-nano-hetero structures able to use nuclear reaction kinematics to self-separate the fission products from the nuclear fuel in microstructures, generically called "Cer-Liq-Mesh", “ 0 " made of nuclear fuel $\left(\mathrm{UO}_{2}, \mathrm{PuO}_{2}, \mathrm{UN}, \mathrm{PuN}\right.$, $\mathrm{PuC}$, etc.) micro-beads, with dimensions inside fission products range, chemically stabilized by a thin coating, soaked in liquid metal fluid. In order to assure mechanical stability the micro-beads are suspended on a wire mesh, or felt that is also elastic. As further analyzed, fission products share about $170 \mathrm{MeV}$ in kinetic energy, and in interaction with the matter surrounding the fission origin they behave like charged particles depositing energy as ionization and nuclear 
recoil towards the end of the stopping range. In solid matter the nuclear recoil zone, also known as Bragg peak is characterized by having many remnant defects as dislocations. The new solid-liquid composite material, places Bragg peak in liquid that has exhibits no remnant structural damage, and the fission product bounds to quid molecules flowing gently outside the area. Figure 2 shows a so called "hydraulic channel", having micrometer size, where the last $10 \%$ of fission product stopping range which is dominated by nuclear recoil takes place in about $1 \mu \mathrm{m}^{3}$ of fluid, depositing the energy there, as seen the left upper chart of Figure 2, upper left side. The temperature field is homogenous drain fluid being a liquid metal, and having larger thermal conductivity than bulky fuel.

This gentle thermal deposition prevents cracks, as they appear in cermet fuels, seen on the right, making possible two new radial temperature deposition curves inside the fuel pellet, that either rises the operating temperature increasing efficiency, or lowering the maximum temperature increasing fuel burnout, or pellet's diameter.

TrOn the bottom charts are presented the advantages of novel micro-hetero-structures comparative to actual fuels, and the left chart shows how power density may be increased by a factor of 4 by using "Cer-Liq-Mesh" structure,


Temperature variation from cladding towards center of the pellet for various materials vs. power density
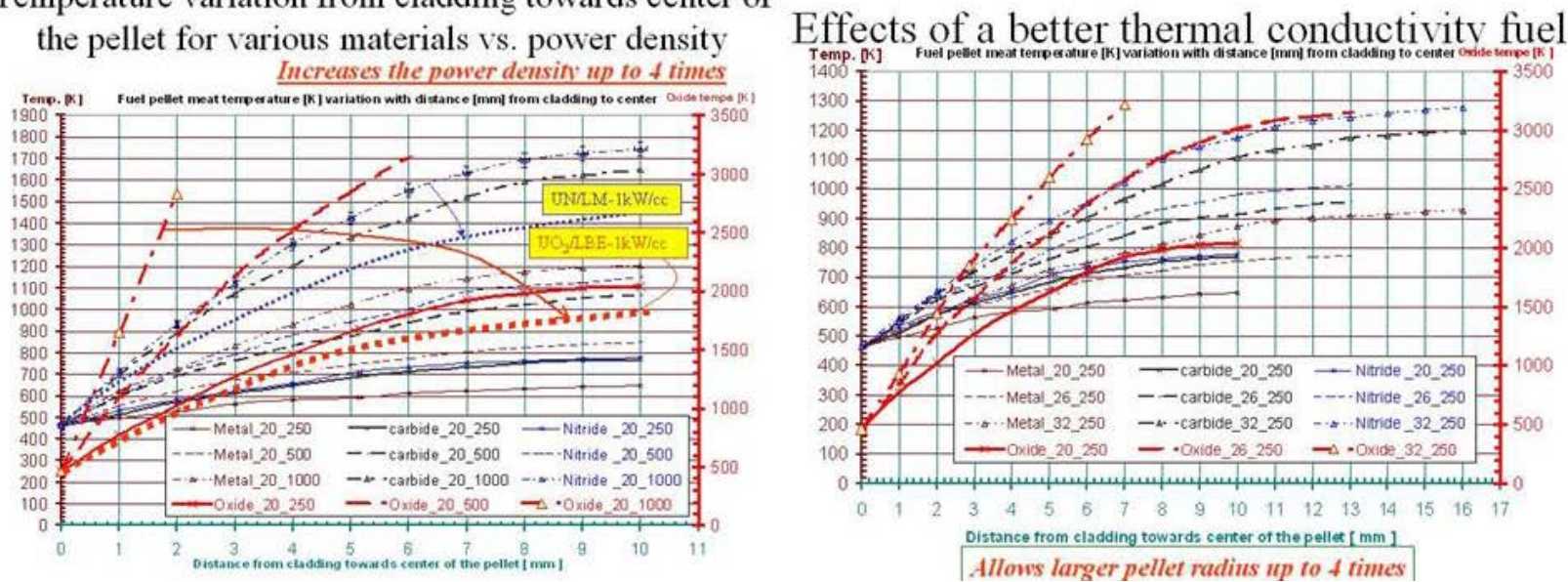

Figure 2. Cer-Liq-Mesh micro-fuel structure and performances. 
or increasing the radial pellet's dimensions by 4 times, simplifying the design and construction, both solution lowering the energy cost. More the solution impacts the fuel cycle, because the fission products are sitting separated from the nuclear fuel, it turns easy to perform fuel refurbishing and recladding for about every $4 \%-10 \%$ of burnup, such as by breed\&burn procedure to burn almost all minor actinides and fertile fuel, with no need for fuel enrichment. The fuel refurbishing uses no harsh chemistry just sequential distillation for separation and partitioning of the fission products, which will be deposited as ore instead of waste.

Having enough elasticity, the fuel may be compressed inside conical reaction channels, to compensate for the lost reactivity due to burnup, and a variant of traveling wave reactor may be built, using $\mathrm{Pu}$ cycle to produce near perfect burnup [2].

These kind of pellets may be produced in short term for the actual PWRs or PHWRs reactors, in order to increase their safety and fuel burnup, being nonproliferation robust, and secure because the fuel refurbishing is done inside the reactor plant in small amounts.

\section{Nano-Beaded-Hetero Structures}

Transmutation products have shorter stopping range in nuclear fuel in $\mathrm{nm}$ range, and in order to extract them, a nano-clustered porous hetero-structure have been developed, where the pores are open and flooded with an extraction fluid, acting as a drain liquid. Nano-cluster exhibit special properties for impurities, different from bulk material that makes a transmutation product created inside a nano-cluster to be expelled on the boundary from where the extraction liquid washes it out. In an ideal case, the micro-bead may be made of a nano-clustered structure contained inside the coating layer together with the extraction liquid, as shown in Figure 3.

This type of nuclear fuel material that contains a porous nano-clustered structure embedded or forming a micro-hetero structure, similar to a frit, that allows the separate extraction of the fission products from the micro-fluid and transmutation products from the nano-fluid may be packed together inside a cladding creating fuel pellet that may be fully compatible and replace the actual nuclear fuel. The advantage is that after a reasonable burnout, the fission or fission and transmutation products may be extracted on spot and the fuel may be reprocessed pellet by pellet, transferred in a new cladding and used in a breed and burn scheme, reducing the need for enrichment, and by this improving the nuclear fuel cycle and nonproliferation resistance. The nano-cluster increased separation capability may be used to extract all kind or transmutation products produced by $n$ irradiation, that are useful to nuclear medicine, industry and radiopharmaceuticals along with minor actinides and super-grade fuel breeding as ${ }^{239} \mathrm{Pu},{ }^{233} \mathrm{U}$ up to ${ }^{251} \mathrm{Cf}$, that may be used in space applications. Figure 3 in the top-right side shows the advantages of using a transport liquid that washes the nano-beads collecting the transmutation products mainly. 


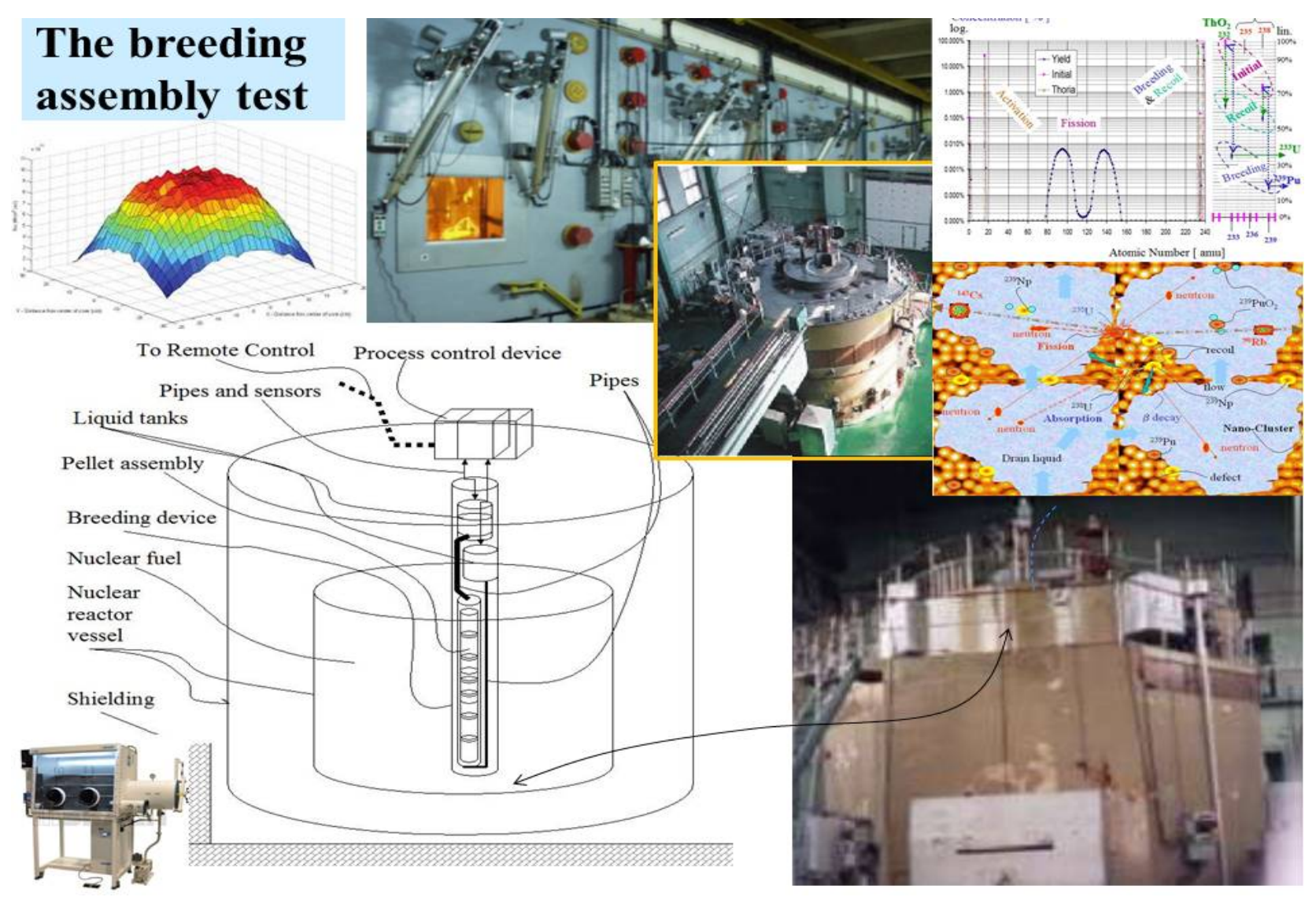

Figure 3. Example of Thorium fuel breeding inside a research nuclear reactor

\section{Nano-Hetero Structure for Direct Nuclear Energy Conversion in Electricity}

Energy released in nuclear reactions is by one million times larger than that delivered in chemical processes, and using engineered nano-hetero structures it become possible to produce battery like systems. There are three types of batteries that can be produced, generically called:

- Isotopic batteries, known for using nuclear transmutation reactions that release alpha or beta radiation, that is harvested and converted into electricity, previously known as alpha or beta voltaic, one such battery delivering the energy of more than 100,000 same power chemical batteries.

- Fission batteries, delivering energy at demand, being in fact a solid-state compact nuclear reactor, where the meta-material inside is harvesting the energy of the fission products, which are over 200 times more energetic than decay reactions, and

- Fusion batteries, where the meta-material is harvesting the energy of the fusion reactors, where fusion is up to three times more energetic than fission. Complementary these meta-materials may be morphed on surfaces, able to convert particle beam energy, useful in space beamed power applications, and being hyperbolic meta-structures for some combinations they exhibit intense EM properties, being possible of emitting $\mathrm{THz}$ up to optical radia- 
tion.

There are many functional configurations of meta-materials that may be used, to convert moving particle energy into electricity as:

- Planar structures, as Figure 4 shows, made of parallel nano-layers of materials, where for harvesting the energy of a 3 micron thick alpha emitter, as ${ }^{210} \mathrm{Po},{ }^{239} \mathrm{Pu},{ }^{241} \mathrm{Am}$, it takes a harvesting double foil of about $50 \mu \mathrm{m}$ thick that may be useful for self-powered electronic modules, or long term batteries. For example using $40 \mathrm{~g}$ of pore ${ }^{238} \mathrm{Pu}$, it may produce a $200 \mathrm{~g}, 40 \mathrm{cc}, 15 \mathrm{~W}$ battery, able to power an artificial heart for more than $40 \mathrm{y}$, or $400 \mathrm{y}$ lifetime batteries for space applications by using ${ }^{241} \mathrm{Am}$. In a modified configuration, the meta-material may work similar to a laser emitting $\mathrm{THz}$ or visible radiation for the same lifetime for data communication purposes.

- Nano-beaded structures, made of a distribution of nano-beads embedded into an amorphous dielectric structure, that have higher operating temperatures and efficiencies than planar structures, and

- Heterogeneous nano-tube structures, believed to exhibit higher conversion efficiencies, over $90 \%$, for isotropic radiation, but exhibiting real constructive difficulties, being now only a theoretical endeavor.

The first products may be isotopic batteries, because require small amounts of nanostructures, producing foils $50 \mu \mathrm{m}$ thick, with a specific power of $1 \mathrm{~mW} / \mathrm{cm}^{2}$ with a $\mathrm{T}_{1 / 2}=87.4 \mathrm{y}$ when using ${ }^{238} \mathrm{Pu}$, or $100 \mathrm{~mW} / \mathrm{cm}^{2}$ with $\mathrm{T}_{1 / 2}=138 \mathrm{~d}$ when using ${ }^{210} \mathrm{Po}$, which may be morphed on the structure. For structures using fission products, $1 \mathrm{~cm}^{3}$ may deliver up to $5 \mathrm{~kW} / \mathrm{mm}^{3}$, but heating is an issue, and in spite the conversion efficiency may be very high, say $90 \%$, at a $1 \mathrm{~kW} / \mathrm{cm}^{3}$ heat flow removal capability, the maximum allowed power density will be of about 10 $\mathrm{W} / \mathrm{mm}^{3}$, by an order of magnitude higher than the maximum actual power densities contained in advanced reactors. In order to be operational, a $1 / 2 \mathrm{~m}$ radius reactor made of nano-structure have to be built in order to reach criticality and this is a problem for the present industry.

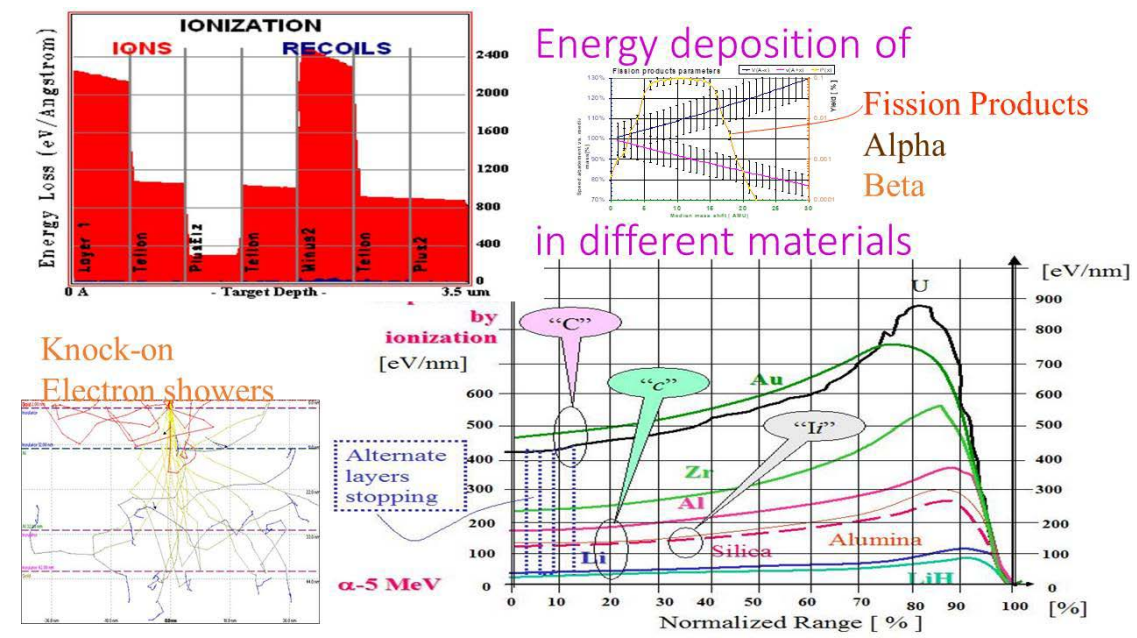

Figure 4. Material selection for "CIci" structure and electron paths and fission products energy and alpha particles energy deposition. 


\section{Radiation Robust Fractal-Hetero-Micro Structures}

In most of the cases, Bragg peak is impossible to be avoided, and the material volume that takes this hit, reaches a local plasma state in a micro volume that cools down and remains with many dpa-s that accumulates and modifies material properties. It is possible to engineer a bi or tri material similar to Damascus steel, but having a fractality of $2.5-2.7$ being made of immiscible interlaced material felts, or solid solutions, which during the recovery from Bragg peak return to previous local equilibrium shape, maintaining constant its properties as function of absorbed radiation dose. By this procedure dislocation density will be maintained at a minimum. Using this material for cladding and structural elements will reduce the need for fuel recladding and allow near perfect burning of the nuclear fuel. The lifetime and safety of such a plant may drastically increase.

\section{Nuclear Radiation Guiding Nano-Structures for Reactivity Control and Shielding Applications}

The present $\mathrm{g}$ ray shielding, relies on mass absorption and is combined with $\mathrm{n}$ shielding while the reactivity control relies on varying $\mathrm{n}$ absorption or $\mathrm{n}$ reflection using outer profiled reflectors, "shadowing" $\mathrm{n}$ absorption blankets, as Figure 5 shows as "classical attenuation". The new process relies on coherent, multiple, small interaction, at the "grazing angle" between a moving particle trapped into a guiding channel and the atoms' force fields forming the channel wall, the particle moving similar to a sleigh on a bob-track.

The scattering is produced by collective quantum levels interaction, where the deflection of moving particle to be under a fraction of a degree per bounce.

\section{Better Shielding + radiation control ${ }^{\text {Nano-waveguides for nuclear }}$}

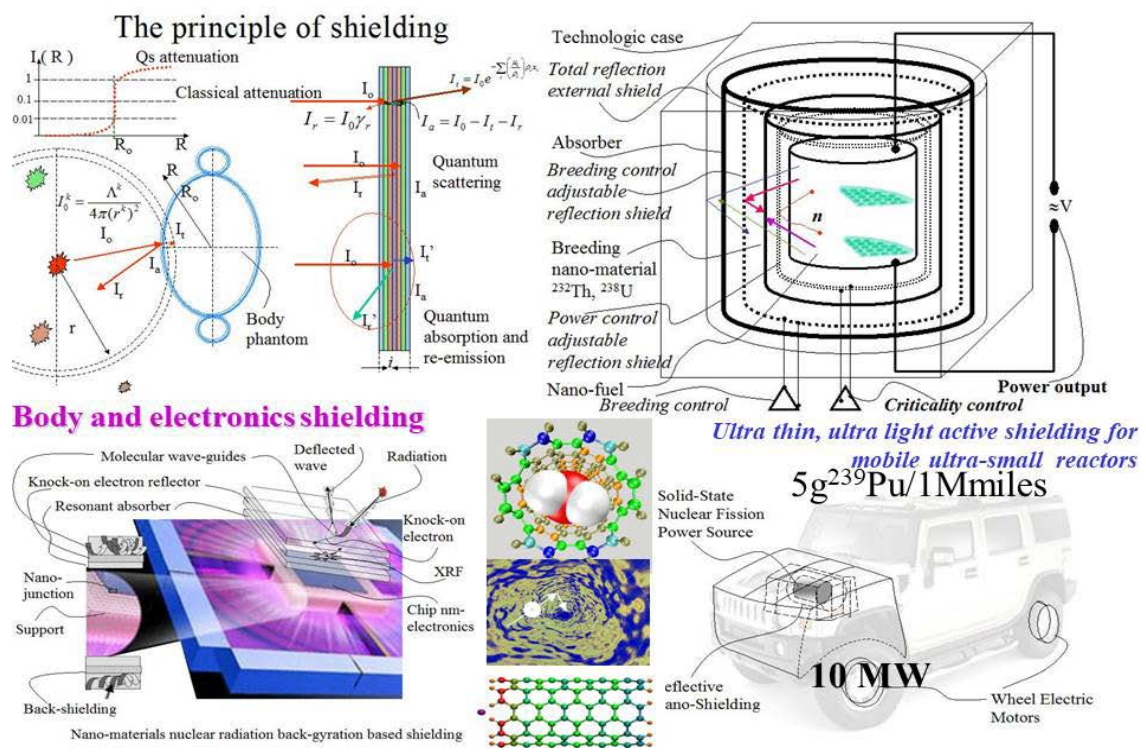

Figure 5. Principle of shielding and few applications. 
For this process one may engineer nano-channels, that may be easily bent, which are able to act as a roundabout for moving particles, changing its direction without absorbing much of its energy, in order to produce radiation structural damage or excessive heating-up. These layers able to harvest radiation coming from a certain cone of incidence and reversing its movement direction are about few $\mathrm{mm}$ thick, and may be built from lighter materials. The addition along the path of electro-sensitive structures acting as switches able to modify the displacement direction of the guided particle by kicking it out of tract may be useful for creating reactivity control systems. A control element for a certain value of the control voltage will allow the particle be turned back into active zone or for another voltage kick particle out of channel and direct it to an absorber zone, reducing the reactivity. This kind of switch may have a rise time in $\mu$ s, having states visible for prompt neutrons, and making possible the whole assembly rise time/ shut-off time in ms. As Figure 5 shows, these materials will permit the building of wearable, high attenuation of radiation, individual protection suits, radiation denial zone over sensitive electronics, to avoid single event upset, or light, thin nuclear reactor shielding, and control systems allowing the construction of a compact, solid-state portable nuclear reactor.

\section{Discussions and Potential Results}

All these materials presented above may be used alone in specific applications in nuclear power, being susceptible of supporting the development of new generations of nuclear reactors, or simultaneous creating hybrid structures with synergistic performances.

The project is in TRL $=3$ stage, having some simulations and ion beam tests accomplished, and more work is needed to develop the highly functional and reliable power sources. It started from the understanding of nuclear reaction kinematics, as Figure 6 shows.

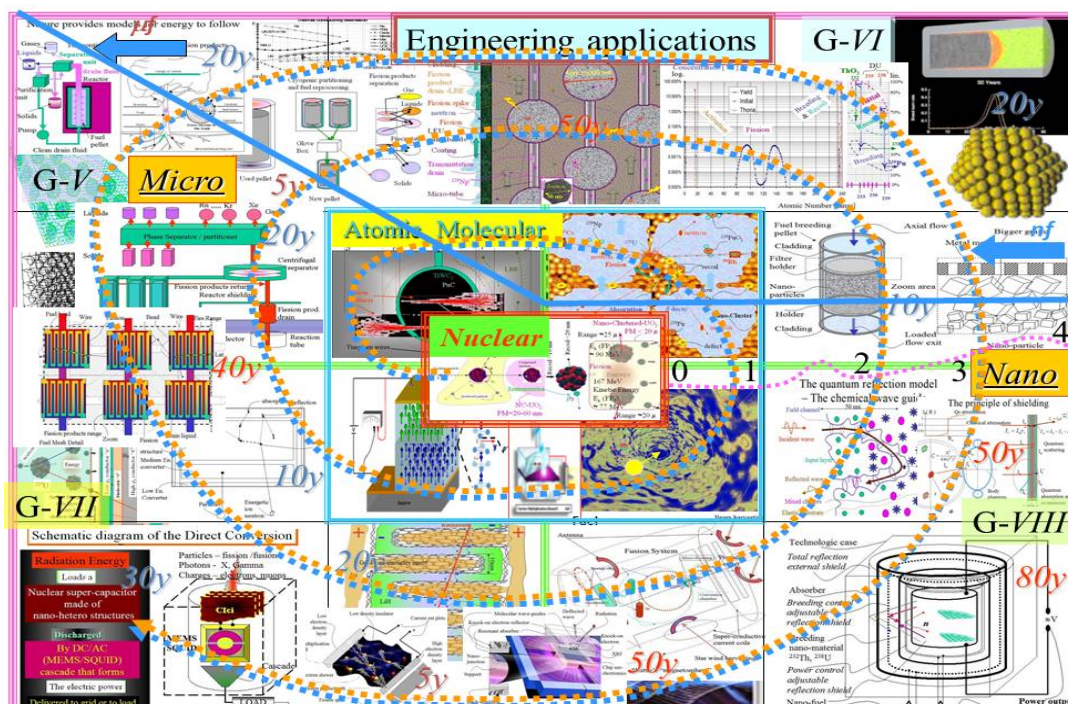

Figure 6. The spiral of evolution from nuclear modeling to engineering applications. 
Atomic and molecular level phase took place in 1980s and drove to incipient formulation of materials tests using ion beams and nuclear reactor in order to prove the feasibility of ideas, and reach TRL $=0$. In late 1990s after level 2 of spiral TRL $=1$ was reached, and was followed by a set of simulations and generic designs for nuclear reactor generations and measurement instruments using the advanced features of micro-nano-hetero structures.

At a minimum dimensions required by criticality conditions, a solid state reactor schematically shown in lower-right side, may have about $1 \mathrm{~m}$ diameter, and few tons, usable similar to a fission battery, suitable to power robust SUVs as seen in Figure 5, and many other applications that require mobile under 1 GW power supplies, being Gen.8 structure.

\section{Conclusion}

The novel, engineered micro-nano-materials will allow the design and constructions of new generations of Nuclear Power plants, and nuclear power related devices, being ecological friendly and assuring unfettered energy for the planet for the next 10,000 years, for terrestrial and space applications, allowing colonization of solar system.

\section{Conflicts of Interest}

The author declares no conflicts of interest regarding the publication of this paper.

\section{References}

[1] Popa-Simil, L. (2015) Accelerated Discovery and Design of Nano-Material Applications in Nuclear Power by Using High Performance Scientific Computing. Research and Applications in Global Supercomputing, Chapter 4-5, 83-148. https://doi.org/10.4018/978-1-4666-7461-5.ch004

[2] Popa-Simil, L. (2008) Micro-Structured Nuclear Fuel and Novel Nuclear Reactor Concepts for Advanced Power Production. Progress in Nuclear Energy, 50, 539-548. https://doi.org/10.1016/j.pnucene.2007.11.041 